\title{
Análisis de Flexo-extensión del Cuello Mediante el Uso de Visión Artificial
}

\author{
Vicente, Kleber $^{1,3, *}$ (D) ; Venegas, William ${ }^{1}$ (D) ; Vásconez, Christian L. ${ }^{2}$ (D) ; Zambrano, Iván $^{1}$ (D) \\ ${ }^{1}$ Escuela Politécnica Nacional, Departamento de Mecánica, Quito, Ecuador \\ ${ }^{2}$ Escuela Politécnica Nacional, Departamento de Física, Quito, Ecuador \\ ${ }^{3}$ Secretaría de Educación Superior, Ciencia, Tecnología e Innovación, Área de Equipamiento EOD-PRETT, Quito, Ecuador
}

\begin{abstract}
Resumen: En el tratamiento del dolor cervical se emplean equipos y técnicas que no miden la intensidad del dolor del paciente, sino que únicamente permiten observar los daños estructurales de dicha región. Sin embargo, la evaluación de éste dolor se puede llevar a cabo al analizar las variables cinemáticas de los tres movimientos de la articulación cervical: flexo-extensión, flexo-lateral y rotación. En este trabajo estudiaremos la fiabilidad de la técnica de fotogrametría, mediante el uso de una cámara de bajo costo, denominado Kinect V1. La cámara Kinect adquirirá los parámetros cinemáticos del movimiento de flexo-extensión de la articulación del cuello y, técnicas de visión artificial y de procesamiento de imágenes de profundidad/color del sensor Kinect serán empleadas para obtener las trayectorias de los marcadores anatómicos y técnicos. Se utilizará un filtro de Kalman para corregir el seguimiento continuo de las trayectorias de los marcadores técnicos y, consecuentemente, las coordenadas espaciales de cada marcador. Los datos fueron obtenidos de siete sujetos de prueba, entre hombres y mujeres, físicamente sanos. Las edades de los sujetos están comprendidas entre 17 y 40 años. Asociados a las coordenadas de cada marcador técnico, calculamos los parámetros cinemáticos de velocidad angular, aceleración angular y desplazamiento angular, para obtener los parámetros de fiabilidad y correlación entre pruebas. Esto último se realizó al analizar el error estándar medio, el índice de correlación múltiple y los índices de correlación de Pearson, empleados para análisis clínico. El alto índice de correlación entre los ensayos realizados nos permite ratificar la fiabilidad de nuestra metodología.
\end{abstract}

Palabras claves: flexo-extensión, fotogrametría, Kinect, filtro de Kalman, cinemática.

\section{Flexo-extension Analysis of the Neck Using Artificial Vision}

\begin{abstract}
In the treatment of cervical pain, several techniques that are commonly used cannot indicate the patient painintensity, but only can identify structural damage. The evaluation of this pain-intensity can be achieved analyzing the cervical joint kinematic variables of the three movements: flexo-extension, flexo-lateral and rotation. In this work we will study the reliability of the photogrammetry technique through a low-cost camera, i.e., Kinect V1. The Kinect camera will acquire kinematic parameters of the flexo-extension movement from the neck joint. We will use artificial vision and depth/color image techniques to obtaining the trajectories of the technical (and anatomical) markers. A Kalman filter is employed to correct the continuous tracking of the technical markers to obtaining the spatial coordinates of each marker. The data is obtained from seven test-subjects, men and women physically healthy. The ages of the testsubjects are between 17 and 40 years. We compute the kinematic parameters of angular velocity, angular acceleration and angular displacement, associated with the spatial-coordinates of each technical marker. Then, we obtain the parameters of reliability and correlation between tests through the mean-standard error, the multiple-correlation index and the Pearsoncorrelation indexes, commonly used for clinical analysis. The high values of these correlation indexes let us to remark the reliability of our methodology.
\end{abstract}

Keywords: flexo-extension, photogrammetry, Kinect, Kalman filter, kinematics.

\section{INTRODUCCIÓN}

\subsection{Antecedentes al estudio del dolor del cuello}

El dolor de cuello (conocido también como columna cervical) es un trastorno musculo-esquelético que afecta a la salud o al normal desenvolvimiento de un individuo. En la actualidad, no se conoce sus precursores exactos y tampoco existen datos epidemiológicos locales (Robaina , 1998; ANT , n.d.). Por ello, las repercusiones a mediano y largo plazo son desconocidas. El sacrificio de la estabilidad del cuello a cambio de la movilidad (Magee , 2013), lo convierte en una zona vulnerable a lesiones, por lo que el estudio de este conjunto de articulaciones es un campo activo. Causas 
subyacentes, como choques del tejido nervioso, patología osteoligamentosa o anormalidades del control neuromuscular (Moskovich , 1988; Pettersson et al. , 1997; Ellingson et al , 2013), han sido identificadas como causas del dolor de cuello. A su vez, estas causas pueden haber sido originadas por traumatismos provocados por accidentes, mala postura, infecciones, condiciones inflamatorias, enfermedades reumáticas o por enfermedades congénitas (Antonaci et al , 2000; De Koning et al. , 2008). La diversidad de precursores para el dolor del cuello dificulta el diagnóstico de la afección, el mismo que depende fuertemente de la metodología empleada para llevarlo a cabo, lo que redunda en un tratamiento inadecuado para tratar la afección (Baydal , 2013). A esta dificultad se le adicionan factores como la estructura anatómica, los movimientos compensatorios, el sexo, la edad, la rutina, entre otros (Antonaci et al , 2000).

\subsection{Técnicas y medidas contemporáneas}

El comportamiento del cuello se puede cuantificar a través del rango de movimiento cervical (cervical range of motion), ROM, cuya medición puede ser obtenida usando métodos invasivos o no invasivos. Entre estos últimos se obtienen datos suficientemente confiables con Rayos X (Williams et al. , 2010), tomografía computarizada y resonancia magnética (Yoganandan et al. , 2001), goniómetros, inclinómetros (Jordan , 2000; Snodgrass et al. , 2014), Cybex o análisis cinemático 3D (Roozmon et al. , 1993) y escáneres electrópticos (Antonaci et al , 2000).

La elección del método depende si el objetivo del mismo será de tipo cribado clínico o con fines investigativos sobre la funcionalidad del cuello. Notamos que el análisis de los movimientos de la columna cervical es más frecuentemente evaluado por motivos clínicos (Antonaci et al , 2000). En este contexto, los métodos más adecuados para una evaluación diagnóstica de la ROM son aquellos basados en inclinómetros, electrogoniometría y video/fotogrametría. Sin embargo, estos métodos son poco repetibles al depender de puntos anatómicos, los mismos que varían entre pacientes (Kraemer and Patris , 1989). Es necesario destacar los estudios realizados por Cescon et al. (2014), que emplean el sistema de detección Virtual Reality Rehabilitation System (VRRS) en Padova, Italia. El equipo VRRS tiene una precisión \pm 0.2 , y sus sensores son colocados en una diadema, fijados con cinta para evitar tracción. En cuanto a las limitaciones de los equipos basados en inclinómetros, destacamos que aunque los movimientos de flexo-extensión y flexión lateral son adecuadamente medidos, las curvas de trayectoria (obtenidas con muestras de movimientos de rotación axial) son del todo no repetibles y, consecuentemente, no fiables (Willinger et al. , 2005).

Por su parte, los equipos de video y fotogrametría garantizan la repetitividad del muestreo, lo que les otorga fiabilidad. En la actualidad, estos sensores son los más empleados para realizar investigación (Baydal , 2013). La técnica de fotogrametría reconstruye coordenadas $3 \mathrm{D}$ a partir de coordenadas $2 \mathrm{D}$, a través de la superposición de imágenes provenientes de al menos dos cámaras. Esto la convierte en una técnica de medición indirecta. Las imágenes 3D son construidas estereoscópicamente por triangulación de puntos homólogos (Sánchez, 2006).
En general, las técnicas empleadas basadas en inclinómetros o goniómetros son poco manejables para los operadores y desagradable para el paciente. Esto repercute negativamente en la fiabilidad de los datos obtenidos y dificulta una evaluación precisa. Entonces, es necesario identificar un método menos invasivo y cuya operación sea menos compleja, sin sacrificar su utilidad clínica (e.g. cámaras digitales). En este documento determinaremos la curva de flexo-extensión del cuello usando equipos de bajo costo como la cámara Kinect de Microsoft. En la Sección 2 explicaremos las características de la cámara Kinect, así como el sistema de referencia que usaremos para los marcadores ópticos. Detallaremos el procesamiento de las imágenes de color y profundidad recolectados y el procedimiento para la adquisión de las coordenadas espaciales. Nuestros resultados experimentales de validación serán resumidos en la Sección 3 y nuestras conclusiones se expondrán en la Sección 4.

\section{Materiales Y Métodos}

\subsection{Sensores ópticos}

La metodología se desarrolla en torno al uso de un sensor Kinect de Microsoft. El dispositivo cuenta con un proyector láser, una cámara infrarroja, una cámara RGB, micrófonos y un procesador personalizado de la marca PRIMESENSEPS1080. Además, el dispositivo tiene una cámara RGB con sensor CMOS (con un filtro de Bayer) con una resolución de $640 \times 480$ pixels, operando a 30fps. Finalmente, tiene una cámara de profundidad basada en un emisor infrarrojo y una cámara infrarroja con sensor CMOS monocromático cuya resolución es de $640 \times 480$ pixels, operando a 30fps. La profundidad de las imágenes se determina en función del tiempo que se demora en reflejar la luz infrarroja (Ladino y Monsalve , 2015; Nuño , 2012). La imagen RGB está compuesta por un conjunto de pixels, compuestos de cuatro componentes: rojo, verde, azul y transparencia (alfa), respectivamente. El último canal funciona también como RGBa y como vacío para imágenes RGB. Cada componente tiene un valor entero de 0 a 254 (correspondiente a byte), por lo que cada pixel posee cuatro bytes. En el caso de la cámara de profundidad, cada pixel almacena un valor de intensidad almacenado en dos bytes, lo que provee 2048 niveles de sensibilidad en profundidad. Éste valor establece la distancia entre el sensor en milímetros (kinectformatlab , n.d.).

\subsection{Marcadores técnicos, anatómicos y de referencia}

Para determinar una adecuada toma de muestra establecemos la ubicación de los marcadores tomando en consideración resultados previos. La cantidad de marcadores varía en cada metodología, aún cuando existen ciertas similitudes entre diversos autores. En particular, podemos resaltar el trabajo realizado recientemente usando técnicas similares de fotogrametría. Baydal (2013) expone la utilización de un casco con cuatro marcadores reflectantes equidistantes en la cabeza. Cada marcador se encuentra a $20 \mathrm{~cm}$ del casco con los objetivos de aumentar la inercia del modelo tipo sólido rígido y disminuir el error en el cálculo de las variables cinemáticas. Para establecer los ejes de referencia locales de cada individuo, se coloca un marcador a la altura de la cervical C7 y dos marcadores situados sobre los lóbulos auditivos. 
Por su parte, Bertomeu y Jaén (2007) usa dos marcadores anatómicos colocados en la espalda (a la altura de la cervical C7), dos marcadores anatómicos (a la altura de los lóbulos auditivos) y tres marcadores técnicos (en la cabeza) empleados durante el movimiento. Díaz-Rodríguez et al (2016) emplea seis marcadores técnicos que se encuentran ubicados en la diadema con cuernos, tres en cada cuerno. Para la etapa de referencia emplea marcadores anatómicos en los lóbulos auditivos y dos en el tórax. La medición de parámetros inerciales se realiza con dos marcadores infraorbitales (ubicados en el punto por debajo de la órbita de los ojos) dos puntos tragiones (aleta de la oreja, sobre el trago) y un marcador Sellion, ubicado en el punto de apoyo de las monturas de las gafas sobre la nariz (McConville et al , 1980).

En el caso de Grip et al. (2007) se utiliza un protocolo con 13 marcadores. Cinco marcadores son colocados en la cabeza, en forma de racimo rígido, un marcador se coloca en la escotadura supraesternal, tres marcadores en una placa rígida en la parte posterior del torso, un marcador en cada hombro y uno en cada fosa mandibular. Los últimos dos marcadores se emplean para estimar la altura cervical y son eliminados antes de la prueba de reposicionamiento. (Grip et al. , 2008) también emplean cinco marcadores en forma de racimo en la cabeza. Además, un marcador en la muesca supraesternal, tres en una placa rígida en la parte posterior, una en cada hombro, una en cada fosa mandibular y cuatro en una placa rígida en cada brazo superior. La placa posterior se la coloca a la altura de las vértebras torácicas T6-T8. Lo que totaliza 21 marcadores, analizados con ProReflex del laboratorio Qualisys Medical AB, utilizando cinco cámaras infrarrojas para un muestreo a $120 \mathrm{~Hz}$.

Ohberg et al. (2003) presentan un tipo de disposición de marcadores diferente: tres marcadores en la cabeza colocadas con unas barras de $11 \mathrm{~cm}$ y separado a $13 \mathrm{~cm}$ uno de otro consecutivamente. En el tórax del paciente se colocó un chaleco ortoplástico con tres marcadores, dos a la altura de la clavícula y uno a la altura de la muesca supraesternal.

La Figura 1 muestra la disposición de los marcadores usados en nuestro estudio. La detección usa cinco marcadores: tres marcadores técnicos ubicados en una diadema a $20 \mathrm{~cm}$ de la cabeza, MT1, MT2 y MT3 (con el fin de aumentar la inercia del modelo del sólido rígido y disminuir el error en el cálculo de las variables cinemáticas, e.g., (Baydal , 2013)). Como marcadores anatómicos, colocamos un marcador a la altura del lóbulo auditivo, MA1, que sirve como un punto de referencia inicial (Díaz-Rodríguez et al, 2016). Así mismo, usamos un punto de referencia en la silla, MS1, para detectar movimientos de la misma en el muestreo.

\subsection{Procedimiento para toma de datos}

Hemos establecido que el número mínimo de sujetos de prueba sean 7 y que el número de muestras por sujeto sean 3, similar al trabajo de Ruíz (2018). Las personas de prueba varían en peso y talla, y sus edades se encuentran entre 17 a 40 años. Todos los sujetos se encuentran aparentemente sanas, sin ningún trauma o enfermedad en las articulaciones del cuello. Se realizan dos muestreos a cada sujeto de prueba en tres sesiones. En el primer caso el paciente no se mueve y se registra las ubicaciones de los cinco marcadores. El segundo muestreo se realiza con

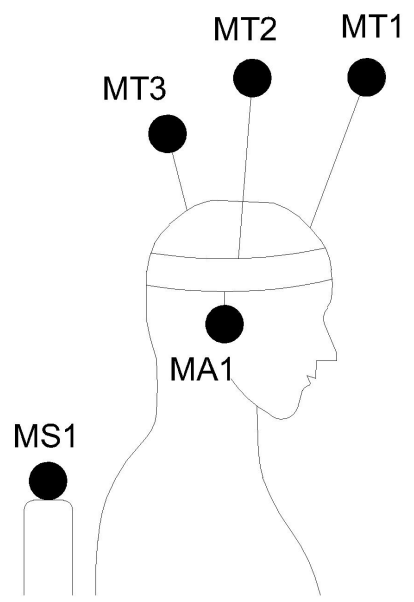

Figura 1. Ubicación de marcadores en el sujeto de prueba. MT1, MT2 MT3 son los marcadores técnicos, colocados a $20 \mathrm{~cm}$ de la cabeza. MA1 es el marcador anatómico, ubicado en el lóbulo auditivo. MS1 es el marcador de referencia de la silla donde se ubica el paciente.

movimientos armónicos y naturales de flexo-extensión para registrar la trayectoria de los tres marcadores técnicos.

La cámara Kinect se coloca a $\sim 1,90 \mathrm{~m}$ desde la pared y a una altura de $\sim 1,05 \mathrm{~m}$. La disposición de la cámara cubre totalmente el campo visual donde se ejecutará la prueba. en peso y talla. El centro de coordenadas $(0,0)$ se obtiene de una imágen de $640 \times 480$ pixels, localizada en $x_{0}=640 / 2$ y $z_{0}=480 / 2$. Gracias al acelerómetro del Kinect podemos controlar por software la inclinación de la cámara. De igual manera, verificamos la perpendicularidad de la cámara al plano de muestreo al comprobar los valores de profundidad con las cuatro esquinas de la habitación donde se toma el muestreo.

\subsection{Adquisición de videos RGB y de profundidad}

La obtención y almacenamiento de las muestras cinemáticas del cuello se llevó a cabo mediante tres programas. El primer programa almacena simultáneamente videos de las imágenes RGB y de profundidad. En este programa hemos tomado las siguientes consideraciones:

- El video para la imagen RGB es almacenado con formato Uncompressed AVI, con extensión “.avi” y con una frecuencia de 30fps.

- La imagen de profundidad se almacena con formato Motion JPEG 2000, con extensión “.mj2”. y con una frecuencia de $30 \mathrm{fps}$. Debemos considerar que cada pixel tiene 16 bits.

El segundo programa procesa los videos para obtener las trayectorias de los marcadores. El programa realiza un procesamiento con visión artificial de la imagen RGB utilizando herramientas de binarizado y operaciones morfológicas, presentes en el programa Matlab. Realizamos este procesamiento en cada frame, tanto de la imagen RGB, como de profundidad. En la Figura 2 podemos apreciar el desfase entre las dos imágenes, propio del arreglo de las cámaras internas del sensor Kinect. En particular, de la imagen RGB obtenemos los valores de las componentes $X$ y $Y$, mientras 
que de la imagen de profundidad obtenemos la componente $Z$.

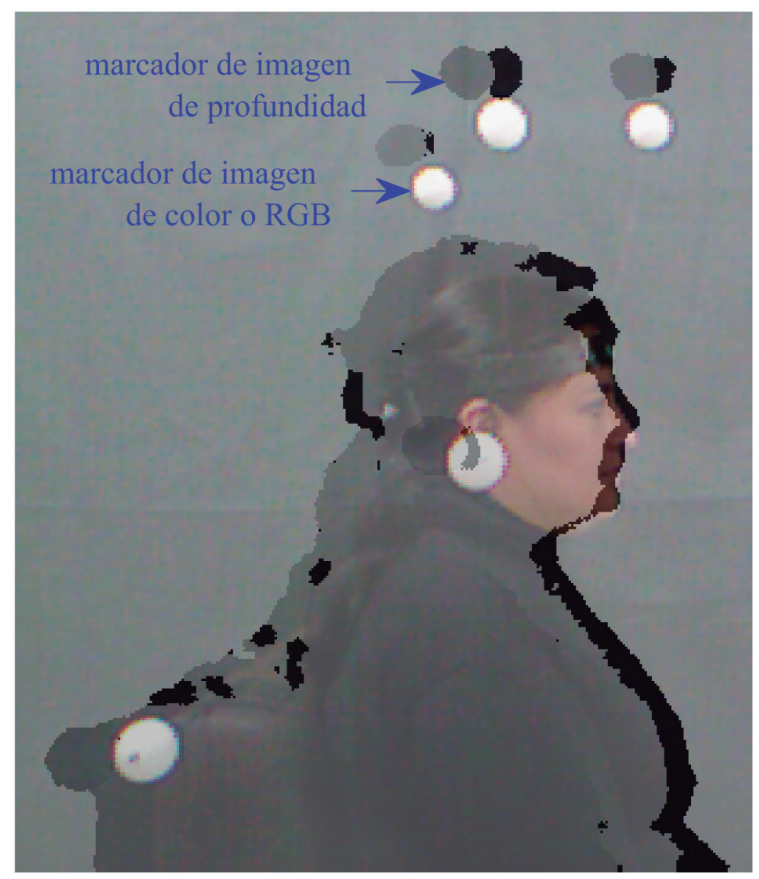

Figura 2. Desplazamiento entre las imágenes de color o RGB (círculos blancos) y de profundidad (círculos gris) causado por la ubicación de las cámaras en el sensor Kinect.

El seguimiento de objetos en movimiento es un tema fundamental dentro de la visión artificial y ampliamente utilizado en muchos campos como la medicina, proyectos militares, vídeo vigilancia, navegación robotizada, entre otros; aunque el seguimiento de múltiples objetos sigue siendo un desafío (Park et al. , 2012; Li et al. , 2010). Para corregir el desplazamiento se utilizó el filtro Kalman, que es un algoritmo que estima el estado del sistema a partir de los datos medidos y luego estimar los estados a partir del error. El filtro permite predecir cuál será la siguiente posición de cada marcador basándose en la posición anterior de cada marcador (Patel y Thakore , 2013), lo que habilita el seguimiento del mismo. Este procedimiento se basa en 1) la predicción del estado del sistema, que se predice con el modelo dinámico y en 2) el paso de corrección, que se corrige con el modelo de observación, de tal modo que el error de la covarianza del estimador es minimizada con un estimador óptimo (Ali y Hassan , 2014; Mathworks , n.d.).

Considerando un sistema de seguimiento, donde $X_{k}$ es el vector de estado que representa el comportamiento dinámico del objeto, nuestro objetivo es estimar $X_{k}$ a partir de la medición de $Z_{k}$, donde el subíndice $k$ indica el tiempo discreto ( $\mathrm{Li}$ et al. , 2010). La descripción matemática se divide en cuatro pasos:

\section{Ecuación del proceso}

$$
x_{k}=\mathbf{A} x_{k-1}+w_{k-1},
$$

donde $A$ representa la matriz de transición y $x_{k}$ el estado al tiempo $k-1$ o $k$, El vector $w_{k-1}$ es el ruido del proceso, con una distribución de probabilidad Gaussiana $p(w) \sim N(0, Q)$.

\section{Ecuación de medición}

$$
z_{k}=H x_{k}+v_{k}
$$

donde $H$ es la matriz de medición y $z_{k}$ es la medida observada al tiempo $k-1$ o $k$, respectivamente, El vector $v_{k}$ es el ruido del proceso, con una distribución de probabilidad Gaussiana $p(v) \sim N(0, R)$.

3. Actualización de ecuaciones del proceso

$$
\begin{gathered}
\hat{x}_{k}^{-}=A \hat{x}_{k-1}+w_{k} ; \\
P_{k}^{-}=A P_{k-1} A^{T}+Q .
\end{gathered}
$$

Con el valor de $z_{k}$, actualizamos los valores desconocidos de $x_{k}$. La estimación del estado a priori $\hat{x}_{k}^{-}$y el error de covariancia $\hat{x}_{k}$ serán obtenidos en el siguiente paso $k$.

\section{Actualización de ecuaciones de medición}

$$
\begin{gathered}
K_{k}=P_{k}^{-} H^{T}\left(H P_{k}^{-} H^{T}+R\right)^{-1} ; \\
\hat{x}_{k}=\hat{x}_{k}^{-}+K_{k}\left(z_{k}-H \hat{x}_{k}^{-}\right) ; \\
P_{k}=\left(1-K_{k} H\right) P_{k}^{-} .
\end{gathered}
$$

El objetivo de este sistema de ecuaciones es la estimación $a$ posteriori a través de $x_{k}$, que es una combinación lineal de la estimación previa y la nueva medición de $z_{k}$. $K_{k}$ es la ganancia de Kalman, que se calcula en función de las ecuaciones de medición actualizadas, después que la estimación de estado $\hat{x}_{k}$ a posteriori y la estimación de error $P_{k}$ a posteriori son calculadas a partir de $z_{k}$. Las ecuaciones de tiempo y de medición se calculan recursivamente con las estimaciones posteriores para predecir una nueva estimación anterior. Este comportamiento recursivo de estimar los estados es uno de los aspectos más destacados del filtro de Kalman (Li et al. , 2010).

Para obtener los centroides de los marcadores en la imagen de profundidad, convertimos a la imagen a una escala de grises y luego la binarizamos tomando en cuenta los umbrales promedio de intensidad, tal como se muestra en la Figura 3. Los umbrales de intensidad se obtienen directamente de la imagen adquirida (en $\sim 11$ capas). En esta escala, los tonos azules son la primera capa y representan los errores de lectura del sensor Kinect, debido a los problemas de reflectancia localizados en los bordes. Los umbrales del 6 al 11 son los más representativos para la lectura de los marcadores y están ordenados como: cyan, verde, amarillo, naranja, naranja-rojizo y rojo. Los umbrales del 6 al 9 contienen a los marcadores y son extraídos empleando operaciones morfológicas. Las capas 1, 10 y 11 son descartadas porque pertenecen a las lecturas erróneas y al fondo de la imagen, respectivamente. Con la obtención de los centroides de los marcadores adquirimos en cada punto la componente $Z$ que nos entregará el valor de profundidad expresado en milímetros. Notamos además que ésta variable tiene formato uint16.

La conversión de unidades de las variables $X, Y$ y $Z$ a centímetros se lleva a cabo tomando en consideración las características de 


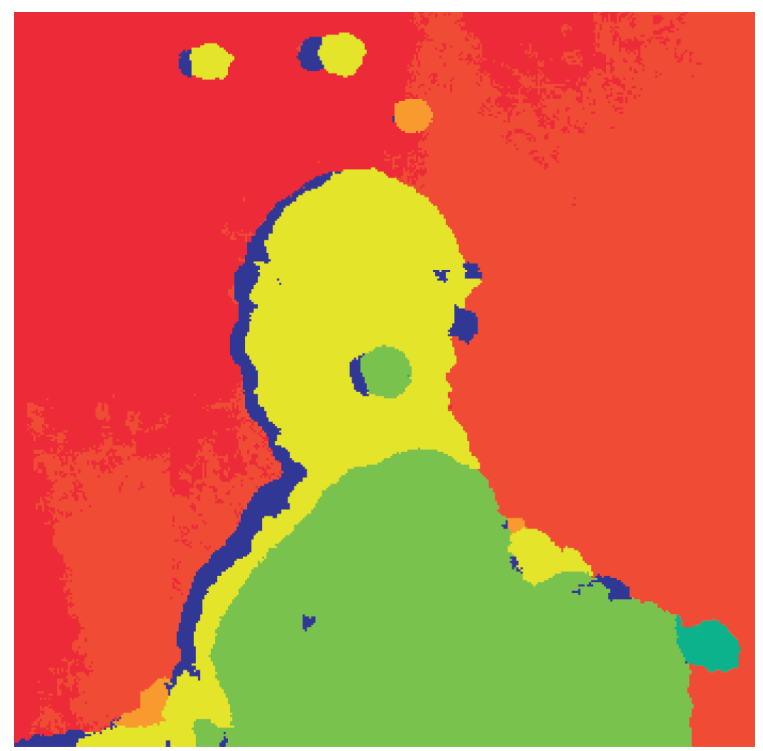

Figura 3. Multi umbrales de intensidad para binarizado de imagen de profundidad. Cada color representa un umbral de intensidad, que nos permite binarizarlo individualmente. Los umbrales de intensidad tienen 11 capas (en promedio). Los tonos azules son la primera capa y representan las lecturas no leídas por el sensor Kinect. Los umbrales del 6 al 11 son los mas representativos para la lectura de los marcadores y están en el siguiente orden: cyan, verde, amarillo, naranja, naranja-rojizo y rojo. Los umbrales cyan, verde, amarillo y naranja contienen a los marcadores que nos proveerán de sus centroides. Hemos descartado las capas 1,10 y 11 .

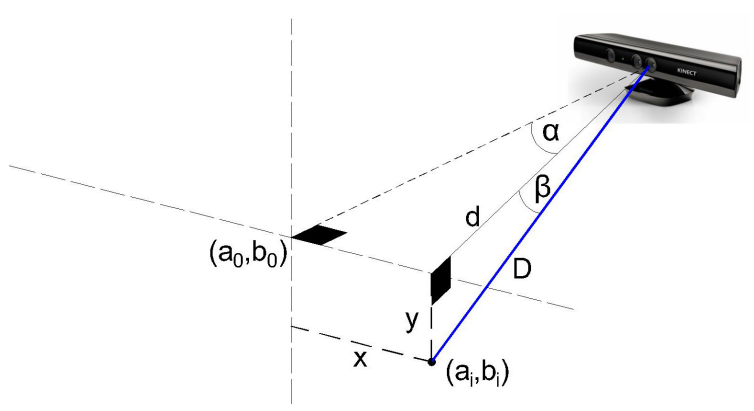

Figura 4. Ubicación del sensor Kinect respecto al plano que contiene las coordenadas espaciales $X-Y$ de los marcadores. Los ángulos de apretura $\alpha$ y $\beta$ son utilizados para convertir de pixeles a centímetros cualquier punto coordenado $\left(a_{i}, b_{i}\right)$, cuyo vector posición es $\mathbf{D}$, y su proyección al eje horizontal $(Y=0)$ es $\mathbf{d}$.

nuestro sensor óptico. La cámara Kinect tiene un ángulo de visión de $57^{\circ}$ en horizontal y $43^{\circ}$ en vertical, con una resolución de $640 \times$ 480 pixels. En nuestro caso de estudio, la disposición geométrica del sensor Kinect se presenta en la Figura 4. El Kinect y la imagen obtenida son perpendiculares por lo que establecemos el centro de la imagen como nuestro punto de referencia $(0,0)$, con lo que $a_{0}=320$ y $b_{0}=240$. En el punto $(0,0)$ los ángulos de apertura $\alpha=\beta=0$ (García y González , 2016). De manera general,

$$
\begin{aligned}
& \beta=\left(b_{0}-b_{i}\right) \frac{43^{\circ}}{480^{\circ} \text { pixel }} ; \\
& \alpha=\left(a_{0}-a_{i}\right) \frac{57^{\circ}}{640^{\circ} \text { pixel }} .
\end{aligned}
$$

Por su parte, las variables $X, Y$ y $Z$ toman en consideración la inversión vertical que se aprecia en la misma Figura 4.

$$
x=-D \sin (\alpha) \cos (\beta) \text {; }
$$

$$
\begin{aligned}
& y=D \sin (\beta) ; \\
& z=D,
\end{aligned}
$$

Donde $D=|\mathbf{D}|$.

\section{RESULTADOS EXPERIMENTALES}

Al observar la Figura 5 apreciamos que las trayectorias de los 3 marcadores técnicos son continuas y se asemejan al movimiento realizado por los sujetos de prueba. La elección del filtro de Kalman resulta ser idónea. Tal como se muestra en la Figura 6, la trayectoria de los marcadores es continua para los 3 componentes y no existe mezcla de datos en el tiempo. Esto podría ocurrir cuando los marcadores se encuentran en el punto de máxima flexión o en el punto de máxima extensión. En los paneles a) y b) se aprecian el punto de máxima extensión en $\sim 25 \mathrm{~s}$ y el punto de máxima flexión en $\sim 11 \mathrm{~s}$, respectivamente, en las componentes $X$ e $Y$. El panel c) evidencia los pequeños desplazamientos en la componente $Z$, cuyos valores pueden interferir en el cálculo de las componentes cinemáticas.

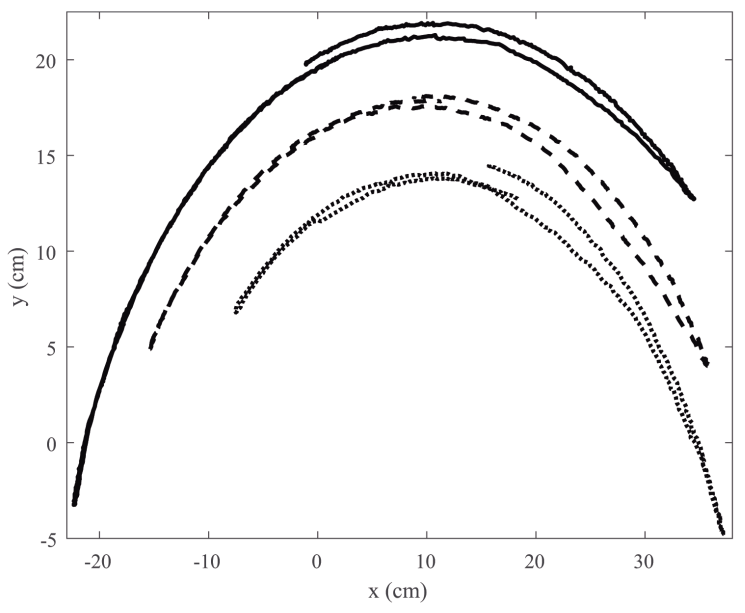

Figura 5. Trayectoria de los tres marcadores técnicos en el plano $X-Y$. La línea continua representa al marcador MT1, la línea segmentada al marcador MT2 y la línea punteada al marcador MT3.

La Tabla 1 recoge las variables cinemáticas más importantes, i.e., ángulo máximo, rango de movimiento (ROM), velocidad angular media $(\bar{\omega})$ y armonía (definida como la pendiente que forma la aceleración angular $\dot{\omega}$ con la posición angular $\phi$ ), obtenidas del análisis de flexo-extensión del cuello para los siete sujetos de prueba y en tres sesiones diferentes.

Tabla 1. Valor medio y desviación estándar (std) de las variables cinemáticas del movimiento de flexo-extensión. Los valores fueron obtenidos de los ensayos realizados a todos los sujetos de prueba, en tres sesiones cada uno.

\begin{tabular}{lcc}
\hline Variable & Media & Std \\
\hline Ángulo máximo $\left(^{\circ}\right)$ & 37.36 & 17.32 \\
ROM $\left(^{\circ}\right)$ & 92.38 & 15.88 \\
$\bar{\omega}\left({ }^{\circ} / \mathrm{s}\right)$ & 0.79 & 1.96 \\
Armonía $\left({ }^{\circ} / \mathrm{s}^{2} /{ }^{\circ}\right)$ & 0.46 & 0.09 \\
\hline
\end{tabular}

La comparación directa de las variables cinemáticas nos muestra que el valor de armonía es inferior a 0.70 , correspondiente a 
trayectorias no lineales y poco reproducibles. Los parámetros de ángulo máximo y ROM tienen alta desviación estándar (Std), lo que confirma que, a pesar de existir buen control en el muestreo, existen parámetros externos que influyen y afectan al sujeto de prueba.
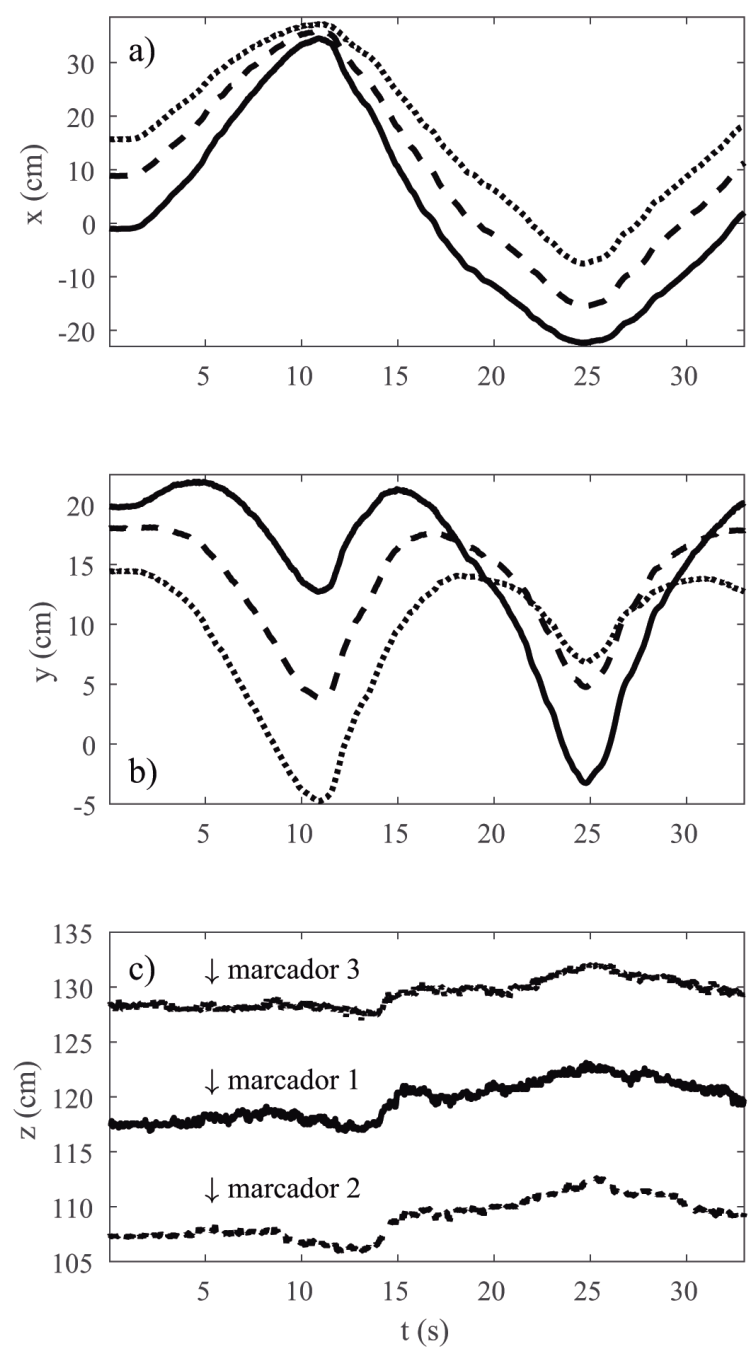

Figura 6. Descomposición $X$ (a), $Y$ (b) y $Z$ (c) de cada trayectoria en función del tiempo $t$. La línea continua representa al marcador MT1, la línea segmentada al marcador MT2 y la línea punteada al marcador MT3.

El análisis de fiabilidad lo realizamos mediante la correlación de las muestras entre sesiones. En nuestro caso, analizamos la correlación entre $\phi$ (o ROM) respecto a $\bar{\omega}$ y a la posición de referencia concerniente al marcador $M A 1(R O)$. Observamos que para el caso de la correlación respecto a $\phi$, el error estándar medio (SEM) es alto, SEM $\in[10.0,18.0]$, pero para la correlación de $\bar{\omega}$ es menor a 7.25 , lo que se puede considerar como un valor aceptable. Los valores de coeficiente de correlación múltiple (CMC) y de Pearson son $\sim 1$. Todo esto indicaría que existe un alto índice de correlación entre las diferentes sesiones (tres por cada individuo). Las Tablas 2, 3, 4 y 5 muestran un alto índice de correlación entre las sesiones y los parámetros cinemáticos de obtenidos de cada sujeto de prueba.
Tabla 2. Valores estadísticos, SEM, CMC y de Pearson, obtenidos de la correlación del ángulo $\phi$.

\begin{tabular}{lccc}
\hline Variable & SEM & CMC & Pearson \\
\hline Sesiones 1 \& 2 & 18.41 & 0.846 & 0.826 \\
Sesiones 1 \& 3 & 14.13 & 0.923 & 0.899 \\
Sesiones 2 \& 3 & 10.32 & 0.848 & 0.930 \\
\hline
\end{tabular}

Tabla 3. Valores estadísticos, SEM, CMC y de Pearson, obtenidos de la correlación de la velocidad angular media $\omega$.

\begin{tabular}{lccc}
\hline Variable & SEM & CMC & Pearson \\
\hline Sesiones 1 \& 2 & 6.61 & 0.872 & 0.845 \\
Sesiones 1 \& 3 & 7.25 & 0.802 & 0.845 \\
Sesiones 2 \& 3 & 4.15 & 0.854 & 0.906 \\
\hline
\end{tabular}

Tabla 4. Valores estadísticos, SEM, CMC y de Pearson, obtenidos de la correlación de RO en función de la posición cartesiana en $X$.

\begin{tabular}{lccc}
\hline Variable & SEM & CMC & Pearson \\
\hline Sesiones 1 \& 2 & 2.52 & 0.832 & 0.898 \\
Sesiones 1 \& 3 & 3.17 & 0.859 & 0.815 \\
Sesiones 2 \& 3 & 1.51 & 0.857 & 0.908 \\
\hline
\end{tabular}

Tabla 5. Valores estadísticos, SEM, CMC y de Pearson, obtenidos de la correlación de RO en función de la posición cartesiana en $Y$.

\begin{tabular}{lccc}
\hline Variable & SEM & CMC & Pearson \\
\hline Sesiones 1 \& 2 & 1.85 & 0.821 & 0.803 \\
Sesiones 1 \& 3 & 3.34 & 0.808 & 0.873 \\
Sesiones 2 \& 3 & 0.84 & 0.823 & 0.857 \\
\hline
\end{tabular}




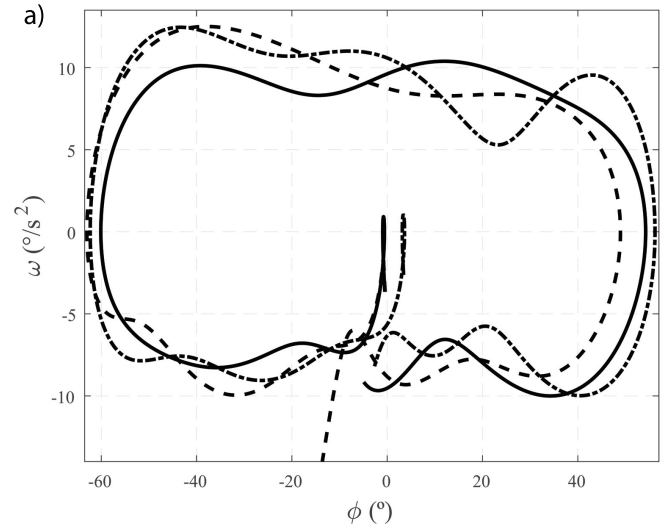

b)

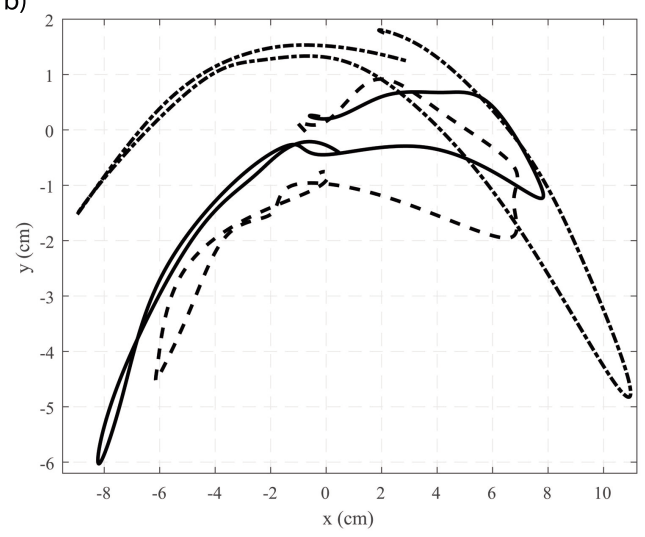

Figura 7. El panel a) se muestra el gráfico fasorial de $\omega$ en función de $\phi$. En el panel b) la curva de desplazamiento suavizada de $x$ en función de $y$, con centro de referencia en el marcador $M A 1$ (ubicado en el lóbulo auditivo). La línea continua representa la sesión 1, la línea segmentada la sesión 2 y la línea punteada la sesión 3.

Las Figuras 7 muestran la evolución de la correlación de $\omega$, en función de $\phi$ para cada uno de las 3 sesiones (panel a)). Se evidencia la no-linealidad del sistema por presencia de factores extrínsecos a las pruebas, y por efecto placebo de los pacientes. En el panel b) observamos la correlación de las variables $X$ y $Y$ al mover el sistema de referencia hacia el marcador MA1 (lóbulo auditivo). Hemos suavizado las curvas por cada sesión. Evidenciamos que el SEM de la posición, respecto a la variable $X$ e $Y$, es pequeño $(<2.70)$. Los valores de coeficiente de correlación múltiple $(\mathrm{CMC})$ y de Pearson son $\sim 1$, lo que indicaría un alto índice de reproducibilidad e implica que la correlación múltiple entre todos los ensayos es elevada. Asimismo, el CMC es alto y cercano a la unidad, lo que implica que la correlación múltiple entre todos los ensayos es elevada. Comparativamente, los valores reportados de SEM, CMC y de Pearson son aceptables. Por ejemplo, Weir (2005) y Schwenk et al. (2012) establecen como un SEM aceptable cuando los valores de intraprueba son similares entre sujetos. Asimismo, Bahat et al. (2014) y Atkinson y Nevill (1998) se refieren al coeficiente de correlación de Pearson como la técnica más común para evaluar la fiabilidad, y consideran valores aceptables del coeficiente cuando es $>0.8$.

Complementariamente, Kadaba et al (1989) sugiere que CMC $>0.8$ es un valor aceptable, basado en la repetibilidad de las sesiones entre sujetos. Notamos que los sujetos 5 y 7 alteran los datos obtenidos (no mostrado aquí), pero a su vez nos permiten validar el experimento con un nuevo equipo, con prestaciones aceptables.

\section{Conclusiones}

La medición de los parámetros cinemáticos del cuello, y su correlación, es de particular valor en la actualidad. Un método que sea confiable y reproducible es necesario.

La técnica presentada en este documento se basa en video y fotogrametría adquiridos con una cámara Kinect. Si consideramos los valores de SEM $(<2)$, CMC $(>0.7)$ e índice de correlación de Pearson $(>0.8)$ de nuestras medidas cinemáticas, podemos concluir que la técnica es fiable.

El analizar la ubicación de los marcadores técnicos, anatómicos y de referencia presentada en este trabajo es importante para la reproducción de la técnica. En particular, la ubicación de marcadores anatómicos como en el lóbulo auditivo son necesarios para aquellos estudios dinámicos porque serviría como referencia para el cálculo de variables, tales como el eje instantáneo de rotación.

Los datos de profundidad obtenidos con el sensor Kinect, y analizados en la Sección 2, mantienen una buena resolución en el rango de los milímetros, pero se ve afectado por los factores de reflectancia de los objetos cercanos, los que producen datos erróneos. Notamos que es probable que éste error se haya corregido con el software y/o hardware del Kinect V2. El panel c) de la Figura 6 evidenció que existen variaciones en el eje $Z$ al momento de adquirir las muestras. Esto nos permitió tomar medidas correctivas para el preprocesamiento de datos.

Al comparar el panel b) de la Figura 7 y la Figura 5, comprobamos que los datos obtenidos poseen una alta correlación para cada sujeto de prueba y entre sesiones. Concluimos que nuestra técnica reproduce adecuadamente la curva de trayectoria del cuello para el movimiento de flexo-extensión. Sin embargo, a pesar de ésta alta correlación, la no-linealidad se debe corregir mejorando el protocolo de medición y capacitando de una mejor manera a los sujetos de prueba para evitar errores por placebo e involuntarios.

La implementación de los filtros de Kalman fue adecuada para el procesamiento de datos en los algoritmos de visión artificial. Estos filtros evitaron la contabilización de falsos positivos cuando se tomaron datos de imagen binarizada.

Finalmente, resaltamos que nuestra técnica implementa un sistema de fotogrametría tridimensional fiable y de bajo costo.

\section{REFERENCIAS}

Ali, N. H., and Hassan, G. M. (2014). Kalman filter tracking. International Journal of Computer Applications, 89(9).

ANT. (n.d.). Estadística de accidentes ANT. Obtenido de: https://www.ant.gob.ec/index.php/descargable/category/79estadisticas. (Marzo, 2019). 
Antonaci, F., Ghirmai, S., Bono, G., and Nappi, G. (2000). Current methods for cervical spine movement evaluation: A review. Clinical and experimental rheumatology, 18(2; SUPP/19), S-45.

Atkinson, G., and Nevill, A. M. (1998). Statistical methods for assessing measurement error (reliability) in variables relevant to sports medicine. Sports medicine, 26(4), 217-238. https://doi.org/10.2165/00007256-199826040-00002.

kinect for matlab (n.d.), Primeros pasos con Kinect. Obtenido de: https://kinectformatlab.es.tl/primerospasosconkinect.htm. (Marzo, 2019).

Ocularis Project. (n.d.). la vision tridimensional. Obtenido de: https://ocularis.es/la-vision-tridimensional/. (Marzo, 2019).

Bahat, H. S., Weiss, P. L. T., Sprecher, E., Krasovsky, A., and Laufer, Y. (2014). Do neck kinematics correlate with pain intensity, neck disability or with fear of motion, Manual therapy, 19(3), 252-258. https://doi.org/10.1016/j.math.2013.10.006

Baydal Bertomeu, J. M. (2013). Cinemática del raquis cervical. Definición de patrones de movimiento para la valoración funcional en el síndrome del latigazo cervical Universidad Politécnica de Valencia, Valencia, España.[PhD Thesis].

Bertomeu, J. M. B., and Jaén, J. D. G. (2007). NedCerv/IBV. Nuevo sistema para la valoración cervical. Revista de biomecánica, 47, 13-16.

Cescon, C., Cattrysse, E., and Barbero, M. (2014). Methodological analysis of finite helical axis behavior in cervical kinematics. Journal of Electromyography and Kinesiology, 24(5), 628-635. https://doi.org/10.1016/j.jelekin.2014.05.004

De Koning, C. H., van den Heuvel, S. P., Staal, J. B., SmitsEngelsman, B. C., and Hendriks, E. J. (2008). Clinimetric evaluation of active range of motion measures in patients with nonspecific neck pain: A systematic review. European Spine Journal, 17(7), 905-921. https://doi.org/10.1007/s00586-008-06563

Díaz-Rodríguez, M., Valera, A., Page, A., Besa, A., and Mata, V. (2016). Dynamic parameter identification of subject-specific body segment parameters using robotics formalism: Case study head complex. Journal of biomechanical engineering, 138(5), 051009. https://doi.org/10.1115/1.4032997

Ellingson, A. M., Yelisetti, V., Schulz, C. A., Bronfort, G., Downing, J., Keefe, D. F., and Nuckley, D. J. (2013). Instantaneous helical axis methodology to identify aberrant neck motion. Clinical Biomechanics, 28(7), 731-735. https://doi.org/10.1016/j.clinbiomech.2013.07.006

García Preciado, P. A., and González Forero, S. N. (2016). Reconocimiento de color y posición con un sensor Kinect para aplicación de robótica móvil, [B.S. thesis]. Facultad de Ingeniería. Pontificia Universidad Javeriana, Bogotá, Colombia.

Grip, H., Sundelin, G., Gerdle, B., and Karlsson, J. S. (2007). Variations in the axis of motion during head repositioning - a comparison of subjects with whiplash-associated disorders or non-specific neck pain and healthy controls. Clinical Biomechanics, 22(8), 865-873. https://doi.org/10.1016/j.clinbiomech.2007.05.008

Grip, H., Sundelin, G., Gerdle, B., and Karlsson, J. S. (2008). Cervical helical axis characteristics and its center of rotation during active head and upper arm movements-Comparisons of whiplash-associated disorders, non-specific neck pain and asymptomatic individuals. Journal of biomechanics, 41(13), 2799-2805. https://doi.org/10.1016/j.jbiomech.2008.07.005

Jordan, K. (2000). Assessment of published reliability studies for cervical spine range -of-motion measurement tools. Journal of manipulative and physiological therapeutics, 23(3), 180-195. https://doi.org/10.1016/S0161-4754(00)90248-3

Kadaba, M., Ramakrishnan, H., Wootten, M., Gainey, J., Gorton, G., and Cochran, G. (1989). Repeatability of kinematic, kinetic, and electromyographic data in normal adult gait. Journal of Orthopaedic Research, 7(6), 849-860. https://doi.org/10.1002/jor.1100070611

Khoshelham, K., and Elberink, S. O. (2012). Accuracy and resolution of kinect depth data for indoor mapping applications. Sensors, 12(2), 1437-1454.

Kraemer, M., and Patris, A. (1989). Radio-functional analysis of the cervical spine using the Arlen method. A study of 699 subjects. Part One: Methodology. Journal of neuroradiology. Journal de neuroradiologie, 16(1), 48-64.

Ladino Agudelo, L. and Monsalve Lesmes, E. (2015). Programa de Procesamiento de Imágenes Adquiridas por Medio el Sensor Kinect para Determinar La Posibilidad una Víctima en Determinada Zona Universidad Distrital Francisco José de Caldas, Bogotá, Colombia. [B.S. thesis].

Li, X., Wang, K., Wang, W., and Li, Y. (2010). A multiple object tracking method using Kalman filter. The 2010 IEEE international conference on information and automation, 1862-1866.

Magee, D. J. (2013). Orthopedic physical assessment. Elsevier Health Sciences.

Mathworks. (n.d.). Diseño y utilización de filtros de Kalman en MATLAB y Simulink. Obtenido de: https://la.mathworks.com/discovery/kalman-filter.html. (mayo, 2018).

McConville, J. T., Clauser, C. E., Churchill, T. D., Cuzzi, J., and Kaleps, I. (1980). Anthropometric relationships of body and body segment moments of inertia. ANTHROPOLOGY RESEARCH PROJECT INC YELLOW SPRINGS OH. Ohio, USA.

Microsoft. (n.d.). Usar las cámaras del sensor. Obtenido de: https://blogs.msdn.microsoft.com/esmsdn/2011/07/20/ reto-kinect-usar-las-cmaras-del-sensor/. (mayo, 2018)

Moskovich, R. (1988). Neck pain in the elderly: Common causes and management. Geriatrics (Basel, Switzerland), 43(4), 65-70. 
Nuño Simón, J. (2012). Reconocimiento de objetos mediante sensor 3D Kinect [B.S. thesis]. Universidad Carlos III, Madrid, España.

Ohberg, F., Grip, H., Wiklund, U., Sterner, Y., Karlsson, J. S., and Gerdle, B. (2003). Chronic whiplash associated disorders and neck movement measurements: An instantaneous helical axis approach. IEEE transactions on information technology in biomedicine, 7(4), 274-282.

Park, S., Yu, S., Kim, J., Kim, S., and Lee, S. (2012). 3D hand tracking using Kalman filter in depth space. EURASIP Journal on Advances in Signal Processing, 2012(1), 36.

Patel, H. A., and Thakore, D. G. (2013). Moving object tracking using kalman filter. International Journal of Computer Science and Mobile Computing, 2(4), 326-332.

Pettersson, K., Hildingsson, C., Toolanen, G., Fagerlund, M., and Björnebrink, J. (1997). Disc pathology after whiplash injury: A prospective magnetic resonance imaging and clinical investigation. Spine, 22(3), 283-287. https://doi.org/10.1097/00007632199702010-00010

Robaina, F. (1998). Cervical whiplash. General features and medicolegal aspects. Rev Soc Esp Dolor, 5, 214-223.

Roozmon, P., Gracovetsky, S., Gouw, G., and Newman, N. (1993). Examining motion in the cervical spine I: imaging systems and measurement techniques. Journal of biomedical engineering, 15(1), 5-12.

Ruíz Cruz, C. A. (2018). Fiabilidad de la técnica de video-análisis en el análisis cinemático de la flexo-extensión del cuello. [B.S. thesis]. Escuela PolitÃ@cnica Nacional, Quito, Ecuador.

Salvatore, J., Osio, J., and Morales, M. (2014). Detección de objetos utilizando el sensor Kinect. Guayaquil, Ecuador, LACCEI.

Sánchez, J. (2006). Introducción a la fotogrametría. ETSI Caminos, canales y Puertos. Cátedra de Topograf'ia, Argentina.

Schwenk, M., Gogulla, S., Englert, S., Czempik, A., and Hauer, K. (2012). Test-retest reliability and minimal detectable change of repeated sit-to-stand analysis using one body fixed sensor in geriatric patients. Physiological measurement, 33(11), 1931. https://doi.org/10.1088/0967-3334/33/11/1931

Snodgrass, S. J., Cleland, J. A., Haskins, R., and Rivett, D. A. (2014). The clinical utility of cervical range of motion in diagnosis, prognosis, and evaluating the effects of manipulation: A systematic review. Physiotherapy, 100(4), 290-304. https://doi.org/10.1016/j.physio.2014.04.007

Venegas-Toro, W., Page Del Pozo, A. F., Zambrano, I., and Ruiz, C. (2018). Análisis del eje instantáneo de rotación del movimiento de flexo-extensión del cuello mediante video análisis: Fiabilidad y análisis de errores. 3C Tecnología, 7(1), 79-92.

Weir, J. P. (2005). Quantifying test-retest reliability using the intraclass correlation coefficient and the SEM. The Journal of Strength and Conditioning Research, 19(1), 231-240. https://doi.org/10.1519/15184.1
Williams, M. A., McCarthy, C. J., Chorti, A., Cooke, M. W., and Gates, S. (2010). A systematic review of reliability and validity studies of methods for measuring active and Passive cervical range of motion. Journal of manipulative and physiological therapeutics, 33(2), 138-155. https://doi.org/10.1016/j.jmpt.2009.12.009

Willinger, R., Bourdet, N., Fischer, R., and Le Gall, F. (2005). Modal analysis of the human neck in vivo as a criterion for crash test dummy evaluation. Journal of sound and vibration, 287(3), 405-431. https://doi.org/10.1016/j.jsv.2004.11.008

Yoganandan, N., Cusick, J. F., Pintar, F. A., and Rao, R. D. (2001). Whiplash injury determination with conventional spine imaging and cryomicrotomy. Spine, 26(22), 2443-2448. https://doi.org/10.1097/00007632-200111150-00010

\section{BIOGRAFÍAS}

Kleber Reynaldo Vicente Romero, Nació en Quito en 1982, Recibió su título de Ingeniero en Electrónica y Control (febrero, 2009) y Magister en Diseño, Producción y Automatización Industrial (diciembre, 2019) en la Escuela Politécnica Nacional (EPN) Quito-Ecuador, Actualmente colabora en el proyecto EOD-PRETT de la Secretaría de Educación Superior, Ciencia, Tecnología e Innovación, su campo de interés es la instrumentación biomédica y biomecánica. Identificador digital ORCID: https://orcid.org/0000-0002-1648-8237

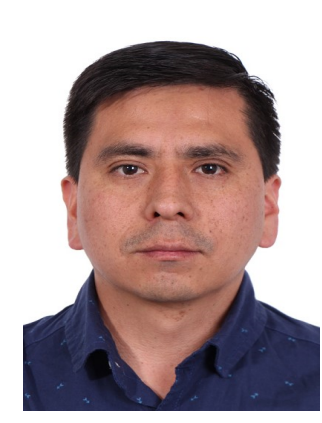

William Ricardo Venegas Toro, is a doctoral student at the Universitat Politécnica of Valéncia (Spain) and an assistant professor at the Escuela Politécnica Nacional (Quito) in the area of computational mehanics and Finite elements. His research is focused on biomechanics models for functional assessment and multiphysical design applications by Finite Elements. Identificador digital ORCID: https://orcid.org/0000-0003-3446-7445

Christian Leonardo Vásconez Vega, Profesor Agregado del Departamento de Física, de la Escuela Politécnica Nacional, desde 2016. Doctor en Física de Sistemas Complejos, con especialización en Plasmas Astrofísicos, por la Universidad de Calabría, Italia. Físico de la Escuela Politécnica Nacional. Del 2007 al 2016, miembro del Observatorio Astronómico de Quito, de la Escuela Politécnica Nacional. Identificador digital ORCID: https://orcid.org/0000-0002-4128-3524 
Iván Zambrano Orejuela, Máster en Métodos Numéricos y Diseño en Ingeniería, Máster en Informática e Ingeniero Mecánico. Actualmente desempeña el cargo de Jefe de Departamento de Ingeniería Mecánica de la Facultad de Ingeniería Mecánica de la Escuela Politécnica Nacional. Sus áreas de interés son diseño y producción, automatización industrial, robótica y mecatrónica. Identificador digital ORCID:

https://orcid.org/0000-0003-3859-7984 